\title{
Factores de riesgo para morbilidad materna extrema en gestantes sin demora en la atención médica según la estrategia camino para la supervivencia
}

\author{
David F. Acelas-Granados ${ }^{1,2}$, Andrea Orostegui ${ }^{1,3}$, Miguel Ángel Alarcón-Nivia. ${ }^{1,4}$ \\ ${ }^{1}$ Universidad Industrial de Santander. ${ }^{2}$ Residente, Programa de Ginecología y Obstetricia, Facultad de Medicina, \\ Universidad Industrial de Santander. ${ }^{3}$ Médico General, Servicio de Urgencias Ginecología y Obstetricia, Hospital \\ Universitario de Santander. ${ }^{4}$ Médico, Ginecólogo Obstetra, Docente Universidad Industrial de Santander. Bucaramanga, \\ Colombia.
}

\section{RESUMEN}

Introducción: La Morbilidad Materna Extrema (MME) es un estado en el cual la gestante casi muere durante su gestación, parto o puerperio pero sobrevivió gracias a una atención oportuna y adecuada, se evalúa por medio de identificación de retrasos o demoras en la atención de la gestante definidos por la Organización Panamericana de la Salud (OPS), los cuales permiten detectar fallas de la paciente o el sistema de salud en su atención, permitiendo mejorar y evitar muertes maternas. Objetivo: Identificar factores de riesgo para MME en gestantes sin demoras en su atención, establecidas según la estrategia "camino para la supervivencia" de la OPS en pacientes atendidas en el Hospital Universitario de Santander (HUS). Método: Estudio de casos y controles de mujeres atendidas en el servicio de urgencias de ginecología y obstetricia del HUS, comparando gestantes con diagnóstico de MME sin demoras OPS en la atención, con gestantes sin diagnóstico de MME en una relación de 1:2, apareadas por número de gestaciones, vía del parto y edad gestacional independiente del trimestre de embarazo o que estuvieran en puerperio. Resultados: 126 pacientes, 42 casos y 84 controles. Atención inicial en sitio inadecuado $(p=0,006)$, nivel educativo bajo $(p=0,011)$ y trastornos hipertensivos en anteriores gestaciones $(p=0,045)$ son factores de riesgo para presentar MME en gestantes sin demoras OPS. Conclusión: Las pacientes con factores de riesgo conocidos deben acudir a instituciones de mayor nivel de complejidad al presentar signos de alarma para evitar incremento del riesgo de eventos de MME.

\section{PALABRAS CLAVE: Morbilidad materna extrema, complicaciones del embarazo, supervivencia}

\section{SUMMARY}

Background: Severe Maternal Morbidity (SMM) is a state in which the mother nearly died during pregnancy, delivery or postpartum but survived thanks to timely and appropriate care, it is assessed by identifying delays or delays in care pregnant women defined by the Pan American Health Organization (PAHO) which detect failures patient or the health system in their care, enabling better and prevent maternal deaths. Objective: To identify risk factors for SMM in pregnant women without delay in their care, according to the strategy established "road to survival" PAHO in patients treated at the University Hospital of Santander (HUS). Method: Case-control study of women treated in the emergency department of obstetrics and gynecology HUS, comparing pregnant women diagnosed with OPS SMM without delay in care, pregnant women without diagnosed with SMM in a ratio of 1:2, paired by number of pregnancies, birth canal and independent gestational age of the trimester or were in postpartum period. Results: 126 patients, 42 cases and 84 
controls. Initial attention in inappropriate site $(p=0.006)$, low educational level $(p=0.011)$ and hypertensive disorders in previous pregnancies $(p=0.045)$ were risk factors in pregnant SMM to present without delay OPS. Conclusion: Patients with known risk factors should attend institutions of higher level of complexity to show signs of alarm to avoid increased risk of events SMM.

\section{KEY WORDS: Severe maternal morbidity, near miss, pregnancy complications, survival}

\section{INTRODUCCIÓN}

Para la mayoría de las mujeres el periodo de gestación y parto se traduce en una experiencia positiva y satisfactoria sin complicaciones; sin embargo, la muerte materna es un desenlace probable si un factor de riesgo no es intervenido oportuna y eficazmente. Dentro de estos extremos se encuentran las condiciones potencialmente peligrosas para la vida o morbilidad materna extrema (MME) la cual puede generar una muerte materna si no es intervenida a tiempo $(1,2,3)$. Se ha calculado que anualmente mueren en el mundo más de 500.000 mujeres por causas relacionadas con el embarazo; de estas muertes, el $99 \%$ se da en países en vías de desarrollo y solo $1 \%$ en países desarrollados $(4,5)$.

La progresión a una muerte materna se relaciona con el tipo de evento, con factores sociales y demográficos, calidad del prestador de servicios de salud y actitud de la paciente hacia el sistema $(6,7,8)$.La Federación Latinoamericana de Sociedades de Ginecobstetricia (FLASOG) y la organización mundial de la salud (OMS) definen la MME como "una complicación severa que ocurre durante el embarazo, parto o dentro de los 42 días posteriores a la terminación del embarazo, pero sobrevive gracias a una intervención médica urgente y oportuna" $(9,10,11)$. Actualmente no existe uniformidad en la definición total de los criterios diagnósticos de MME los cuales cambian de acuerdo a la región geográfica y la disponibilidad de recursos sanitarios de cada país. Sin embargo, en Colombia, para definirla se exige la presencia de al menos una de las tres directrices propuestas por el por el Instituto Nacional de Salud (INS) y FLASOG (Figura 1) $(2,12-15)$. Existe una gran ventaja en la posibilidad de analizar el caso de una mujer que sobrevivió a complicaciones potencialmente mortales durante el embarazo parto o puerperio, al reconocer los obstáculos y demoras para recibir atención adecuada desde los niveles clínicos básicos, la falta de recursos locales, pobreza, desventajas sociales correspondientes y los posibles retrasos en el proceso de referencia (16-20). Por tal motivo un método utilizado con el propósito de evaluar y lograr mejoramiento en la atención de la paciente gestante se denomina "camino para la supervivencia" (CS), adoptado por la OMS y el Fondo de Población de las Naciones Unidas.
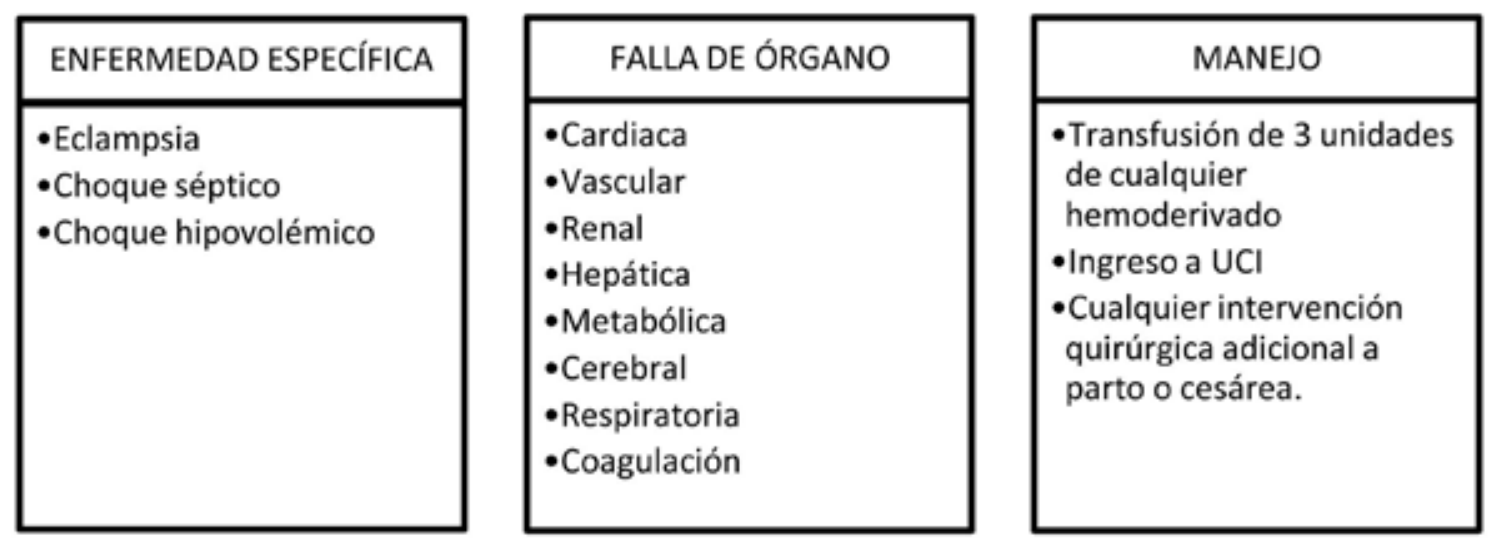

Figura 1. Criterios diagnósticos de Morbilidad Materna Extrema. 
El CS se basa en el supuesto de que para reducir la MME no es suficiente que los servicios de salud funcionen eficientemente sino en las barreras que generan retrasos a las pacientes para poder acceder a ellos (Figura 2) $(19,20,21)$. Es claro que para disminuir la mortalidad materna se deben focalizar las intervenciones en la categoría de MME; sin embargo, la incidencia de MME en Colombia está en ascenso con 14.010 casos para Noviembre de 2015 (22), de acuerdo a esto, se han publicado trabajos sobre MME en Cartagena en 2008 y Medellín en $2014(23,24)$ estableciendo que aún existen altas tasas de mortalidad materna asociada a episodios de MME con retrasos de diversa índole, embarazos no deseados y deficiencias en el control prenatal.

Un estudio realizado en el Hospital Universitario de Santander (HUS) entre 2009 y 2011 reportó que el $25 \%$ de todos los casos con MME no presentó ninguna demora en la atención según la evaluación realizada de acuerdo a los retrasos establecidos por la OPS, por lo cual según estos resultados, serían episodios de MME no prevenibles (25). Acorde a estos datos, existe el interrogante sobre la presencia de factores de riesgo aun no identificados en el desarrollo de escenarios asociados a MME.

El objetivo de este estudio observacional es precisar si existen factores de riesgo adicionales en pacientes con MME sin presencia de demoras según el CS que puedan ser previstos para evitar estas complicaciones durante la gestación parto o puerperio.

\section{MATERIALES Y METODOS}

EI HUS es un hospital público de tercer nivel con manejo de pacientes de alta complejidad, centro de referencia del departamento de Santander con atención a población obstétrica de alto riesgo, predominantemente afiliada al régimen subsidiado de seguridad social o no asegurada. Se realizó un estudio de casos y controles con una muestra de mujeres gestantes atendidas en el servicio de urgencias de ginecología y obstetricia del HUS. Se comparó a pacientes gestantes con criterios diagnósticos de MME definidos por el INS-FLASOG y ausencia de retrasos OPS en su atención, de acuerdo al análisis realizado por el comité de MME del HUS, las cuales fueron definidas como casos. Las pacientes definidas como controles

\section{RETRASO TIPO 1}

No reconocimiento del problema (signos de peligro) a

\section{RETRASO TIPO 2
Dificultades en la toma de decisiones oportunas sobre buscar ayuda y atención ${ }^{b}$}

\section{RETRASO TIPO 3}

No acceso a una atención oportuna ${ }^{c}$

\section{RETRASO TIPO 4 No recibir atención de calidad ${ }^{\mathrm{d}}$}

Figura 2. Retrasos en la atención oportuna y adecuada de una gestante según la clasificación de la Organización Panamericana de la Salud.

a Falta de información de la gestante sobre posibles complicaciones del embarazo. Desconocimiento de y derechos en salud sexual y reproductiva.

${ }^{b}$ Barreras sociales, culturales o económicas o experiencias previas negativas con servicios de salud que retrasan la búsqueda de atención por parte de la gestante.

${ }^{C}$ Retraso relacionado con vías, transporte, sistemas de remisión de pacientes entre la comunidad y el personal sanitario.

d Eventos que ocasionan atención deficiente en los servicios de salud tanto acto médico como labores administrativas inadecuadas. 
fueron gestantes sin criterios diagnósticos de MME, con igual edad gestacional que los casos, la misma vía de finalización de la gestación o en el escenario de que el caso continuara la gestación el control también fue una paciente que continuo la gestación e igual número de gestaciones que la paciente caso. Estos criterios de apareamiento fueron tenidos en cuenta ya que las patologías cambian de acuerdo al trimestre de embarazo. La cesárea representa aumento de la morbilidad sobre el parto vaginal, y a mayor número de gestaciones existe incremento de riesgo de complicaciones en la gestación, con el fin de hacer comparables las 2 poblaciones de estudio. Se tuvo una relación de 1 caso con 2 controles. Las pacientes ingresaron al estudio independiente del trimestre de embarazo o si se encontraban en puerperio, entre los años 2013 y 2014. Los controles fueron seleccionados secuencialmente por conveniencia teniendo en cuenta las dos siguientes pacientes gestantes atendidas en el servicio de urgencias de ginecología y obstetricia del HUS, que cumplieran criterios de control posterior a la fecha de presentación del caso correspondiente. De acuerdo a la prevalencia local disponible de MME sin retrasos, el tamaño de muestra fue de 126 pacientes correspondientes a 42 casos y 84 controles. Se excluyeron casos de mortalidad materna, gestantes con criterios de MME y presencia de retrasos en la atención documentados por el comité de MME del HUS y casos no analizados por el comité de MME del HUS. Las pacientes casos y controles fueron identificadas de los registros de datos manuales de ingreso al servicio de urgencias de ginecología y obstetricia, sala de partos del HUS y las fuentes de información fueron las historias clínicas electrónicas institucionales.

Todas las pacientes fueron analizadas y clasificadas por el comité Institucional de MME conformado por ginecólogos, perinatólogos, especialistas en salud pública, epidemiología y trabajo social, adscritos como trabajadores del HUS y docentes de la Universidad Industrial de Santander. Las variables analizadas fueron sitio y nivel de atención inicial, remisión, edad, municipio de residencia, nivel educativo, régimen de salud, ocupación, estado civil, paridad, ausencia o presencia de control prenatal, trimestre de inicio del control prenatal, número de controles prenatales, desenlace del recién nacido, antecedentes maternos, familiares, obstétricos y no obstétricos, hemoclasificación materna, primipaternidad y estrato socioeconómico de la paciente. Para el análisis de las variables cuantitativas se presentan con su respectiva medida de dispersión desviación estándar o percentiles (p 25-75) según la distribución de los datos, las variables cualitativas se expresan como frecuencias absolutas y relativas. La base de datos se elaboró en el programa Excel, con ingreso de datos semanal y los análisis se realizaron en el programa Stata 13.1. El protocolo contó con la aprobación del comité de ética del HUS así como del comité de ética en investigación científica de la Universidad Industrial de Santander. Se consideró significativa una diferencia con valor $\mathrm{p}<0,05$.

\section{RESULTADOS}

En la base de datos se analizaron 126 pacientes que cumplían criterios de inclusión las características de los grupos fueron comparables (Tabla I). El $88 \%$ de los casos de MME se presentaron en el tercer trimestre y el $97 \%$ fueron gestaciones simples. Las principales causas de MME de acuerdo al presente estudio se observan en la (Figura 3). El $66 \%$ de los casos presentó 3 o más criterios, y los más frecuentes para definir MME fueron falla en la coagulación con un $57 \%$ e ingreso a UCI con $55 \%$ de casos. El $86 \%$ de los casos ingresó remitida al HUS, en un $59 \%$ de municipios del área metropolitana de Bucaramanga, y de estos, $45 \%$ provenían de instituciones de primer nivel de atención. El $66 \%$ de los casos terminaron la gestación con un peso promedio de recién nacido de 2.375 gramos y el $88 \%$ egresaron vivos de la institución.

La atención inicial en institución de salud no adecuada se asoció con presencia de MME en pacientes sin demoras (OR 4,00; IC95\% 1,24-14,01), sin que fuera posible discriminar por ciudad de remisión ni por nivel de complejidad de la institución remisora. No terminar la secundaria fue factor de riesgo para presentar MME en pacientes sin demoras (OR 3,33; IC95\% 1,08-10,97). Tener antecedente de trastornos hipertensivos en anteriores gestaciones (THAE) presentó asociación positiva para MME sin demoras (OR13,6; IC95\% 1,8-124,85). A su vez presentar eventos de MME está asociado a pérdidas gestacionales tempranas antes de las 28 semanas de gestación con fetos menores a 1000 gramos (Tabla II). No se encontró asociación entre tener un neonato menor de 2.500 gramos con MME (OR 3,33; IC95\% 0,97-11,83). Antecedentes familiares como THAE y patología psiquiátrica mostraron tendencia al riesgo para presentar MME sin demoras pero con relación estadísticamente no significativa. El haber terminado secundaria es un factor protector para MME (OR 0,30; IC95\% 0,09$0,93)$. En cuanto a las variables restantes ninguna fue estadísticamente significativa. 
Tabla I

CARACTERÍSTICAS DE LOS GRUPOS

\begin{tabular}{|c|c|c|}
\hline Variable & $\begin{array}{l}\text { Casos } \\
(n=42)\end{array}$ & $\begin{array}{c}\text { Controles } \\
(\mathrm{n}=84)\end{array}$ \\
\hline \multicolumn{3}{|l|}{ Lugar de remisión: } \\
\hline Bucaramanga & $12(29 \%)$ & $14(17 \%)$ \\
\hline Floridablanca & $3(7 \%)$ & $4(5 \%)$ \\
\hline Girón & $3(7 \%)$ & $4(5 \%)$ \\
\hline Piedecuesta & $3(7 \%)$ & - \\
\hline Otros municipios & 15 (36\%) & 25 (30\%) \\
\hline No remitida & $6(14 \%)$ & $37(44 \%)$ \\
\hline \multicolumn{3}{|c|}{ Nivel institución de remisión: } \\
\hline I nivel & $19(45 \%)$ & $24(29 \%)$ \\
\hline II-III nivel & $17(40 \%)$ & $23(28 \%)$ \\
\hline Ninguna & $6(14 \%)$ & $37(44 \%)$ \\
\hline \multicolumn{3}{|l|}{ Edad materna: } \\
\hline Mediana (RIQ) & 22 (19 a 25) & $23(19$ a 29.5$)$ \\
\hline \multicolumn{3}{|l|}{ Municipio de residencia: } \\
\hline Bucaramanga & $14(33 \%)$ & $27(32 \%)$ \\
\hline Floridablanca & $2(5 \%)$ & $6(7 \%)$ \\
\hline Girón & $4(10 \%)$ & $11(13 \%)$ \\
\hline Piedecuesta & $4(10 \%)$ & $4(5 \%)$ \\
\hline Otros municipios & $18(43 \%)$ & $36(42 \%)$ \\
\hline \multicolumn{3}{|l|}{ Vivienda: } \\
\hline Urbana & $31(74 \%)$ & $52(62 \%)$ \\
\hline Rural & $11(26 \%)$ & $32(38 \%)$ \\
\hline \multicolumn{3}{|l|}{ Régimen seguridad social: } \\
\hline Subsidiado & $36(86 \%)$ & $71(85 \%)$ \\
\hline Contributivo & $4(10 \%)$ & $4(5 \%)$ \\
\hline Prepagada & 0 & 0 \\
\hline Vinculado/sisben & $2(5 \%)$ & $7(8 \%)$ \\
\hline Particular & 0 & $2(2 \%)$ \\
\hline Especial & 0 & 0 \\
\hline \multicolumn{3}{|l|}{ Estrato social: } \\
\hline $1-2$ & $28(67 \%)$ & $56(67 \%)$ \\
\hline $3-4$ & $14(33 \%)$ & $28(33 \%)$ \\
\hline $5-6$ & 0 & 0 \\
\hline \multicolumn{3}{|l|}{ Estado civil: } \\
\hline Unión libre & $21(50 \%)$ & $43(51 \%)$ \\
\hline Casada & $6(14 \%)$ & $5(6 \%)$ \\
\hline Viuda & 0 & 0 \\
\hline Divorciada/separada & 0 & $1(1 \%)$ \\
\hline Soltera & $11(26 \%)$ & $27(32 \%)$ \\
\hline \multicolumn{3}{|l|}{ Nivel educativo: } \\
\hline Analfabetismo & 0 & $2(2 \%)$ \\
\hline Básica primaria & $9(21 \%)$ & 25 (30\%) \\
\hline Secundaria incompleta & 23 (55\%) & $18(21 \%)$ \\
\hline Secundaria completa & $3(7 \%)$ & $22(26 \%)$ \\
\hline Técnico/universitario & $4(10 \%)$ & $5(6 \%)$ \\
\hline No definido & $3(7 \%)$ & $12(14 \%)$ \\
\hline
\end{tabular}




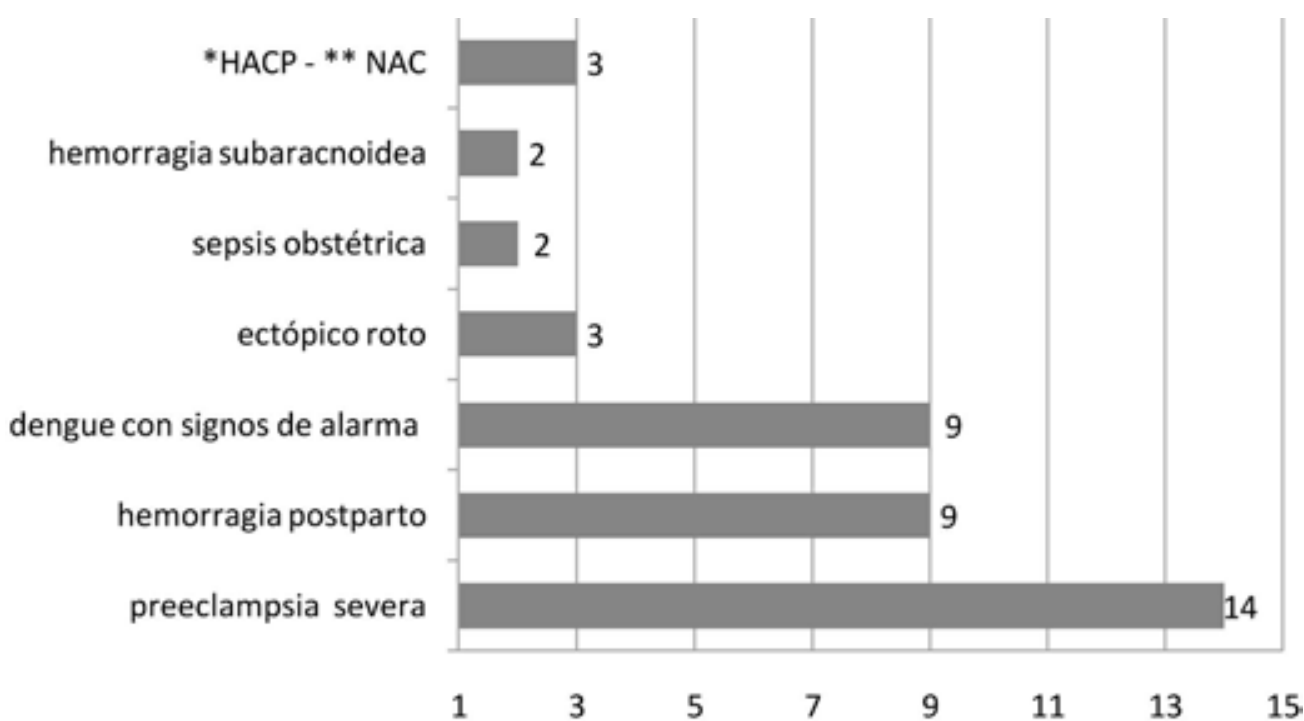

Figura 3. Principales causas de Morbilidad Materna Extrema (MME).

HACP - NAC: herida por arma corto punzante - neumonía adquirida en la comunidad.

Tabla II

FACTORES DE RIESGO PARA PRESENCIA DE MORBILIDAD MATERNA EXTREMA SIN DEMORAS

\begin{tabular}{lcc}
\hline Variable & Valor $p$ & OR (IC95\%) \\
\hline Ingreso remitida & 0,001 & $4,00(1,24-14,01)$ \\
No terminar bachillerato & 0,029 & $3,33(1,08-10,97)$ \\
THAE & 0,045 & $13,6(1,8-124.85)$ \\
Pérdida gestacional temprana & 0,004 & $2,37(1,12-13,4)$ \\
Peso <2.500 gramos & $\mathrm{NS}$ & $3,31(0,97-11,83)$ \\
Antecedentes familiares & $\mathrm{NS}$ & $1,85(0,72-9,33)$ \\
Terminar bachillerato & 0,019 & $0,30(0,09-0,93)$ \\
\hline
\end{tabular}

NS: no significativo. THAE: Trastornos hipertensivos en anteriores embarazos.

\section{DISCUSIÓN}

El presente estudio buscó posibles factores de riesgo para desarrollar MME en una subpoblación de gestantes sin demoras detectadas en su atención o manejo, las cuales fueron definidas previamente por la OPS de acuerdo a la estrategia camino para la supervivencia. Los trastornos hipertensivos y hemorrágicos siguen siendo las primeras causas de MME y las manifestaciones derivadas de estos como falla en la coagulación e ingreso en la $\mathrm{UCI}$ son los principales criterios para definir un episodio de MME en Colombia $(23,26,27)$.
La atención inicial de una paciente en institución de salud no adecuada con retraso en su traslado a una institución de mayor complejidad con capacidad para manejo de pacientes gestantes causa un incremento del riesgo de presentar MME, hallazgos acordes con Hirose y cols en 2015, en estudio llevado a cabo en hospitales de segundo y tercer nivel de la ciudad de Herat en Afganistán (28). El bajo nivel educativo y los antecedentes de complicaciones por THAE fueron factor de riesgo para MME, de igual forma el hecho de terminar la etapa de básica secundaria se presenta como un factor protector para presentar MME lo cual es 
acorde con lo descrito por Lindquist y cols (29) y Assarag y cols (30).

Dentro de los desenlaces estadísticamente significativos se encuentra también el bajo peso fetal al momento de terminar la gestación, lo cual indica que la presencia de MME es un factor de riesgo para recién nacidos con peso bajo para su edad gestacional (4). Estratos socioeconómicos bajos, desempleo de la gestante, tabaquismo o paridad no tuvieron significancia estadística como factor de riesgo para MME en este estudio, lo cual se podría explicar por posibles resultados debidos al azar secundarios al tamaño de muestra. Sin embargo, estudios como el realizado por Lindquist en 2013 reportan datos similares en estas asociaciones de la paciente con riesgo de presentar MME (29). A pesar de la no significancia estadística, sí es destacable la relevancia clínica de estos datos puesto que más del $80 \%$ de los casos provienen de estratos 1 y 2 y pertenecen al régimen subsidiado, siendo esto un marcador de la persistencia de inequidades sociales que hace que este tipo de población tenga bajo acceso a los servicios de salud en Colombia. De acuerdo a esto, se requiere un estudio con un tamaño de muestra mayor en el cual se pueda inferir otros factores de riesgo con un poder epidemiológico mayor. El hecho de ser un estudio realizado en una sola institución, de carácter retrospectivo, en el que los datos se obtuvieron de las historias clínicas puede constituir una limitación ya que estos registros pueden tener fallos en su información por ausencia u omisión de los mismos.

\section{CONCLUSIÓN}

El análisis de las demoras convencionales, no cubre de manera total las falencias y los posibles factores de riesgo para presentar MME, por lo cual se debe adicionar al análisis de retrasos en cualquier paciente con MME la valoración del nivel educativo, permanencia prolongada en el sitio de atención inicial, no correspondiente con el riesgo materno respectivo y los antecedentes obstétricos previos relacionados con trastornos hipertensivos como posibles retrasos, con el fin de incidir sobre ellos y de esta forma disminuir el porcentaje de pacientes con MME teóricamente inevitables. Así mismo se debe crear una historia clínica obstétrica unificada con una clasificación sencilla y ágil de las variables trazadoras de riesgo evitando la progresión a casos de MME. Hay que incrementar actividades de mejoramiento en el control prenatal con el fin de educar a la paciente y a su entorno familiar acerca de los posibles signos de alarma en el inicio de un caso de MME, con las indicaciones necesarias para consultar de manera rápida a una institución con la capacidad adecuada de atención de su patología y no presentar demoras en el inicio oportuno de un adecuado manejo en instituciones de menor complejidad.

\section{REFERENCIAS}

1. Geller SE, Rosenberg D, Cox SM, Brown ML, Simonson L, Driscoll CA, et al. The continuum of maternal morbidity and mortality: Factors associated with severity. Am J Obstet Gynecol. 2004;191:939-44.

2. Say L, Souza JP, Pattinson RC. Maternal near miss - towards a standard tool for monitoring quality of maternal health care. Best Pract Res Clin Obstet Gynaecol. 2009;23:287-96.

3. Cecatti JG, Souza JP, Neto AFO, Parpinelli MA, Sousa MH, Say L, et al. Prevalidation of the WHO organ dysfunction based criteria for identification of maternal near miss. Reprod Health [Internet]. BioMed Central Ltd 2011;8(1):22.

4. Instituto Nacional de Salud, Minsalud. República de Colombia. Protocolo de vigilancia en Salud Pública. Morbilidad Materna Extrema. PRO-R02.052 Versión 02, 29 de Marzo 2016. Disponible en: www.ins.gov. c0./lineas-de-acción/Subdireccion-Vigilancia/sivigila/ Protocolos\%20SIVIGILA/PRO\%20MORBILIDAD\%20 MATERNA\%20Extrema.pdf.

5. WHO, Unicef, UNFPA and The World Bank. Trends in Maternal Mortality: 1990 to 2008 . Disponible en: apps. who.int/iris/bitstream/10665/44423/1/9789241500265_ eng.pdf.

6. Geller SE, Rosenberg D, Cox SM, Brown ML, Simonson $\mathrm{L}$, Driscoll CA, et al. The continuum of maternal morbidity and mortality: Factors associated with severity. Am J Obstet Gynecol. 2004;191:939-44.

7. Pacheco AJ, Katz L, Souza AS, de Amorim MM. Factors associated with severe maternal morbidity and near miss in the São Francisco Valley, Brazil: a retrospective, cohort study. BMC Pregnancy Childbirth. 2014;14:91.

8. Jabir M, Abdul-Salam I, Suheil DM, Al-Hilli W, AbulHassan S, Al-Zuheiri A, et al. Maternal near miss and quality of maternal health care in Baghdad, Iraq. BMC Pregnancy Childbirth. 2013;13:11.

9. Kayem G, Kurinczuk J, Lewis G, Golightly S, Brocklehurst $P$, Knight $M$. Risk factors for progression from severe maternal morbidity to death: a national cohort study. PLoS One. 2011;6:e29077.

10. Ministerio de la Protección Social. República de Colombia. Plan de choque para la reducción de la mortalidad materna. Bogotá, 2003. Disponible en: www.0ssyr.org.ar/pdf/bibliografia/5.2.pdf.

11. Ministerio de la Protección Social. República de Colombia. Vigilancia epidemiológica de la morbilidad materna extrema. Una nueva estrategia para la reducción de la mortalidad materna en América Latina. Fondo Población las Naciones Unidas (UNFPA), 2013. Disponible en: www.who.int.pmnch/ events/2010/20100809_colombia.pdf.

12. Ps R, Verma S, Rai L, Kumar P, Pai M V., Shetty J. "Near miss" obstetric events and maternal deaths in a tertiary care hospital: An audit. J Pregnancy. 2013;2013:393758.

13. Jarquim D, Estrada F, Ortiz E, Marín, Montufar C. [Severe acute maternal morbidity (near miss) for severe preeclampsia in Central América]. Revista 
Centroamericana de Obstetricia y Ginecología 2010;15(1 supl 1):41-68. Disponible en: file:///C:/Users/ DAVID\%20ACELAS/Downloads/ suplementoparte2. pdf.

14. Instituto Nacional de Salud, Ministerio de Salud y Proteccion Social. Republica de Colombia. Bogota: INS Protocolo de vigilancia en Salud Pública. Morbilidad Materna Extrema. 2014;36.

15. Lobato G, Nakamura-Pereira M, Mendes-Silva W, Dias MB, Reichenheim ME. Comparing different diagnostic approaches to severe maternal morbidity and near-miss: A pilot study in a Brazilian tertiary hospital. Eur J Obstet Gynecol Reprod Biol; 2013;167:24-8.

16. Cecatti JG, Souza JP, Parpinelli MA, De Sousa MH, Amaral E. Research on severe maternal morbidities and near-misses in Brazil: what we have learned. Reprod Health Matters 2007;15(30):125-33.

17. Soni-Trinidad C, Gutiérrez-Mateos A, Santa RosaMoreno FJ, Reyes-Aguilar A. Morbilidad y mortalidad materna y factores de riesgo asociados con una urgencia obstétrica [Maternal mortality and morbidity and risk factors associated with an obstetric emergency]. Ginecol Obstet Mex 2015;83(2):96-103.

18. González Ortiz LDG, Gómez Arias RD, Vélez Álvarez GA, Agudelo Londoño SM, Gómez Dávila J, Wylie J. [Characteristics of hospital care and its relationship to severe maternal morbidity in Medellín, Colombia]. Rev Panam Salud Publica. 2014;35:15-22.

19. WHO. Beyond the numbers: reviewing maternal deaths and complications to make pregnancy safer. $\mathrm{Br}$ Med Bull. 2004;67(830):27-37.

20. Pacagnella RC, Cecatti JG, Parpinelli MA, Sousa MH Haddad SM, Costa ML, et al. Delays in receiving obstetric care and poor maternal outcomes: results from a national multicentre cross-sectional study. BMC Pregnancy Childbirth. 2014;14:159.

21. Thaddeus S, Maine D. Too far to walk: Maternal mortality in context. Soc Sci Med. 1994;38(8):1091-110.

22. Instituto Nacional de Salud [pagina principal en internet].Bogota: [actualizada Mayo 2016; consultada 15
Diciembre de 2015]. Contenido Colombia vigila. Notificación semana 48;2015. Disponible en: http://www. ins.gov.co/boletin-epidemiologico/Boletn\%20Epidemiolgico/2015\%20Boletin\%20epidemiologico\%20semana\%2048.pdf.

23. Bello Alvarez L, Vásquez Dielafoaut D, Rojas Suárez $\mathrm{JA}$, et al. Extreme maternal morbidity indicators university clinic in third level of complexity. A retrospective evaluations. Rev Cienc Biomed 2012; 3(2):291-9.

24. González Ortiz LDG, Gómez Arias RD, Vélez Álvarez GA, Agudelo Londoño SM, Gómez Dávila J, Wylie J. [Characteristics of hospital care and its relationship to severe maternal morbidity in Medellín, Colombia]. Rev Panam Salud Publica 2014;35(1):15-22.

25. Andrea N, Arguello A. Caracterización de la Mortalidad y Morbilidad Materna extrema en el Hospital Universitario de Santander [Tesis]. Bucaramanga: Universidad Industrial de Santander. Facultad de Salud; 2011.

26. Ronsmans C. Severe acute maternal morbidity in lowincome countries. Best Pract Res Clin Obstet Gynaecol. 2009;23(3):305-16.

27. Madeiro AP, Rufino AC, Lacerda ÉZG, Brasil LG. Incidence and determinants of severe maternal morbidity: a transversal study in a referral hospital in Teresina, Piaui, Brazil. BMC Pregnancy Childbirth. BMC Pregnancy and Childbirth. 2015;15:210.

28. Hirose A, Borchert M, Cox J, Alkozai A, Filippi V. Determinants of delays in travelling to an emergency obstetric care facility in Herat, Afghanistan: an analysis of cross-sectional survey data and spatial modelling. BMC Pregnancy Childbirth. 2015;15:14.

29. Lindquist A, Knight M, Kurinczuk JJ. Variation in severe maternal morbidity according to socioeconomic position: a UK national case-control study. BMJ Open. 2013;1-8.

30. Assarag B, Dujardin B, Delamou A, Meski F. Determinants of Maternal Near-Miss in Morocco: Too Late, Too Far , Too Sloppy ? PLoS One. 2015;10:115. 